\title{
Data Mining Method for Comparative Analysis of Personal Loans Based on Principal Component Analysis, Naive Bayes and Decision Tree
}

\author{
Menuka Maharjan \\ Assistant Professor, Computer Engineering Department, Nepal Engineering College, Bhaktapur, Kathmandu, Nepal \\ Menukam@nec.edu.np
}

\begin{abstract}
The data mining classification techniques and analysis can enable banks to move precisely classify consumers into various credit risk group. Knowing what risk group a consumer falls into would allows a bank to fine tune its lending policies by recognizing high risk groups of consumers to whom loans should not be issued, and identifying safer loans that should be issued on terms commensurate with the risk of default. So research en for classification and prediction of loan grants. The attributes are determined that have greatest effect in the loan grants. For this purpose C4.5, CART and Nä̈ve Bayes are compared and analyzed in this research. This concludes that a bank should not only target the rich customers for granting loan but it should assess the other attributes of a customer as well which play a very important part in credit granting decisions and predicting the loan defaulters.
\end{abstract}

Keywords: C4.5, CART, Naïve Bayes, Type II error

\section{Introduction}

The decision-making of accepting or rejecting a client's credit by banks is commonly executed via judgmental techniques and credit scoring models. Most banks and financial institutions use the judgmental approach which is based on the 3C's, 4C's or 5C's which are character, capital, collateral, capacity and condition. However, to improve assessment of credit applicants, banks can use credit scoring or predictive models to classify the applicants[1].A bank loans officer needs analysis of his/her data in order to learn which loan applicants are safe" and which are risky" for the bank.To understand that information, classification is a form of data analysis that can be used to extract models describing important data classes or to predict future data trends. Several classification techniques have been proposed over the years e.g., neural networks, genetic algorithms, Naive Bayesian approach, decision trees, nearest-neighbour method etc [2].

The classification is dependent on characteristics of the borrower (such as age, education level, occupation, marital status and income), the repayment performance on previous loans and the type of loan. In this study, my attention is restricted to C4.5,CART and Naïve Bayes classification considering its advantages like efficiency with respect to time accuracy, data, etc and analyze different parameters (age,income,credit rating job etc.) those influence the loan grants.

\section{Methodology}

Classification is learning a function that maps an item into one of a set of predefined classes. It is the type of data analysis that can be used to extract models to describe important data classes or to predict future data trends. The classification process consists of two phases; the first phase is learning process, the training data will be analyzed by the classification algorithm. The learned model or classifier is represented in the form of classification rules. Next, the second phase is classification where the test data are used to estimate the accuracy of the Classification model or classifier. If the accuracy is considered acceptable, the rules can be applied to the classification of new data [3].This section is about the framework for comparing the performance of the classification algorithms of decision trees: CART,C4.5 and Naïve Bayes classification with the role play of the attributes in them to predict loan grants data is taken from data sets[9]. It consists of 1000 data, among which $60 \%$ are used for training and remaining $40 \%$ are utilized for testing purpose that are work

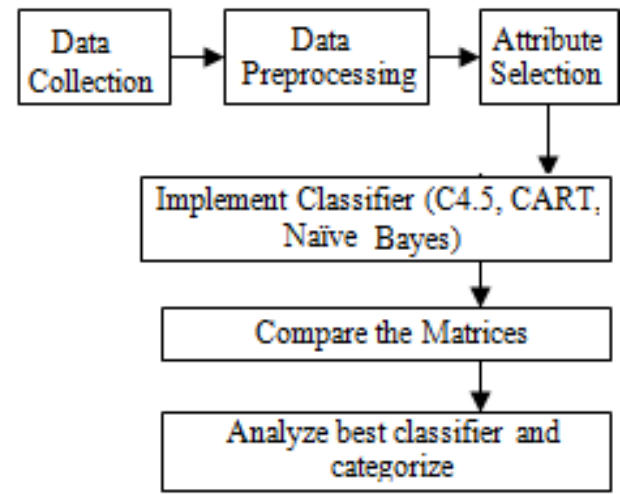

Figure: Research Methodology

\section{Loan Prediction using C4.5}

C4.5 is an algorithm used to generate a decision tree developed by Ross Quinlan. C4.5 is an extension of Quinlan's earlier ID3 algorithm. The decision trees generated by $\mathrm{C} 4.5$ can be used for classification, and for this reason, $\mathrm{C} 4.5$ is often referred to as a statistical classifier [7].

\section{C4.5 algorithm:}

For the classification the total number of good and bad in loan grants is found out from the data set. Information gain is calculated for the whole dataset i.e. Info (D) and then for each attribute the normalized information gain is calculated individually i.e. Info(D) .Gain(A) is calculated subtracting 
the information gain and information gain of individual attribute for that particular attribute.

$$
\begin{gathered}
\mathrm{IG}(\mathrm{A})=\mathrm{H}(\mathrm{S})-\sum_{t \in T} p(t) H(t) \\
\text { Where, }
\end{gathered}
$$

$\mathrm{H}(\mathrm{S})$ - Entropy of set $S$, and $\mathrm{H}(\mathrm{S})=-\sum_{x \in X} p(x) \log _{2} p(x)$

$\mathrm{T}$ - The subsets created from splitting set $\mathrm{S}$ by attribute $\mathrm{A}$ such that

$\mathrm{P}(\mathrm{T})$ - The proportion of the number of elements in $t$ to the number of elements in set $S$

$\mathrm{H}(\mathrm{t})$ - Entropy of subset $t$

The process is repeated for all the attributes and selected the highest normalized information gain for a decision node. The features of the attribute may be nominal or categorical like if age is attribute with its category like youth, middleaged and senior. For each category the table with the remaining attributes is made. Again, recursion is done until leaf node is not found.

\section{Loan Prediction Using Cart}

Classification and regression trees (CART) is a nonparametric decision tree learning technique that produces either classification or regression trees, depending on whether the dependent variable is categorical or numeric, respectively.

\section{CART algorithm:}

It will search for all possible variables and all possible values in order to find the best split - the question that splits the data into two parts with maximum homogeneity. The process is then repeated for each of the resulting data fragments which use impurity functions like Gini splitting index and Towing splitting index [6]. Here Gini splitting rule (or Gini index) is used for the loan prediction. It uses the following impurity function:

\section{Splitting Criteria:}

Gini index is measured to find the impurity of $D$, a data partition or set of training tuples, as

$$
\operatorname{Gini}(\mathrm{D})=1-\sum_{i=1}^{m} P i^{2}
$$

where $p i$ is the proabability that a tuple in $D$ belongs to class $C i$. The sum is computed over $m$ classes.

Here, splitting is compulsory binary so, data $D$ is splittedinto $D 1$ and $D 2$. The partitioning is done as follows

$$
\operatorname{Gini}_{\mathrm{A}}(\mathrm{D})=\frac{|D 1|}{|D|} \operatorname{Gini}(\mathrm{D} 1)+\frac{|D 2|}{|D|} \operatorname{Gini}(\mathrm{D} 2)
$$

The reduction in impurity that would be incurred by a binary split on a discrete or continuous-valued attribute $A$ is

$$
\Delta \operatorname{Gini}(\mathrm{A})=\operatorname{Gini}(\mathrm{D})-\operatorname{Gini}_{\mathrm{A}}(\mathrm{D})
$$

The process is repeated for each attributes and decision for the rootnode is made for the lowest valued $\operatorname{Gini}_{\mathrm{A}}(\mathrm{D})[6]$. Again if the attribute purpose is chosen as the root node then its features like personal loan and business loan is splitting binary and made the table for only each features in both sides.Recursion is done until leaf node is found.

\section{Loan Prediction using Naive Bayes}

A Naïve Bay's classifier estimates the class-conditional probability by assuming that the attributes are conditionally independent, given the class label $y$. The conditional independence assumption can be formally stated as follows:

$$
P(X \mid Y=y)=\prod_{i=1}^{d} P(X i \mid Y=y),
$$

Where each attribute set $\mathrm{X}=\left\{\mathrm{X}_{1}, \mathrm{X} 2 \ldots \mathrm{X}_{\mathrm{d}}\right\}$ consists of $d$ attributes. [8 ]"

\section{Algorithm}

1) From data set $D$ Associated class label $n$ dimensional attribute vector $\mathrm{X}=(\mathrm{x} 1, \mathrm{x} 2, \mathrm{x} 3, \ldots, \mathrm{xn})$, depiction $\mathrm{n}$ measurement made on the tuple from $n$ attributes. A1, A2, A3... An

2) Suppose we have $m$ classes $c 1, c 2, \ldots, \mathrm{cm}$ Giving tuple $\mathrm{X}$, classifier will predict $\mathrm{X}$ belongs to highest posterior probability, condition on $\mathrm{X}$.

$\mathrm{X} \in \mathrm{Ci}$ if $\mathrm{P}(\mathrm{Ci} \mid \mathrm{X})>\mathrm{P}(\mathrm{Cj} \mid \mathrm{X})$ for $1<=\mathrm{j}<=\mathrm{m}, \mathrm{j} \mid \mathrm{Ci}$, for which $\mathrm{P}(\mathrm{Ci} \mid \mathrm{X})$ is maximized is called maximum posterior hypothesis;

$P(C i \mid X)=\frac{P(X \mid C i) P(C i)}{P(X)}$

3) $\mathrm{P}(\mathrm{X})$ is constant for all classes maximize $\mathrm{P}(\mathrm{X} \mid \mathrm{Ci}) \mathrm{P}$ (Ci).

If Class prior probability are not known, commonly assume that $\mathrm{P}(\mathrm{C} 1)=\mathrm{P}(\mathrm{C} 2)=\ldots=\mathrm{P}(\mathrm{Cm})$ maximize $\mathrm{P}(\mathrm{X} \mid \mathrm{Ci})$

Else Maximize $\mathrm{P}(\mathrm{X} \mid \mathrm{Ci}) \mathrm{P}(\mathrm{Ci})$

i. $\quad P\left(C_{i}\right)=\frac{|C i, D|}{|D|}$

4) Calculate $\mathrm{P}(\mathrm{X} \mid \mathrm{Ci})$ is extremely expensive Naïve assumes class conditional independence is made.

$P\left(X \mid C_{i}\right)=\prod_{k=1}^{n} P(X k \mid C i)$, $=P(X i \mid C i) . P\left(X_{2} \mid C i\right) \ldots P(X n \mid C i)$

Where $X_{k}$ is the value of attribute, $A_{k}$ for $X$.

If $\mathrm{A}$ is category

$\mathrm{P}\left(\mathrm{X}_{\mathrm{k}} \mid \mathrm{C}_{\mathrm{i}}\right)=\frac{\# o f_{-} \text {tuple_of_class_Ci_inD_have_value_Xk }}{|C i, D|}$

\section{Results and Discussion}

The German loan dataset consist of 1000 dataset $60 \%$ of data is used for the train set and $40 \%$ is used for the test set. Experiments for CART and C4.5 using German data set are summarized below:

Table 1: C4.5 train

\begin{tabular}{|l|c|c|c|c|c|c|c|c|c|c|c|c|}
\hline \multirow{2}{*}{ Attributes } & \multicolumn{3}{|c|}{ Confusion Matrix } & \multirow{2}{*}{ Precision } & \multirow{2}{*}{ Recall } & \multirow{2}{*}{ F_Score } & \multirow{2}{*}{ Accuracy } & \multirow{2}{*}{ CCI } & Time & \multirow{2}{*}{ No. of Leaf } & \multirow{2}{*}{ Size of Tree } \\
\cline { 2 - 16 } & TP & FN & FP & TN & & & & & & \\
\hline category1 & 398 & 25 & 67 & 110 & 85.5914 & 94.08983 & 89.63964 & 398.18333 & 508 & 0.2 & 52 & 75 \\
\hline category2 & 404 & 19 & 54 & 123 & 88.20961 & 95.50827 & 91.71396 & 404.205 & 527 & 0.1 & 7 & 13 \\
\hline category3 & 398 & 25 & 67 & 110 & 85.5914 & 94.08983 & 89.63964 & 398.18333 & 508 & 0.2 & 52 & 75 \\
\hline category4 & 394 & 29 & 110 & 67 & 78.1746 & 93.14421 & 85.00539 & 394.11167 & 461 & 0 & 16 & 23 \\
\hline category5 & 359 & 64 & 70 & 107 & 83.68298 & 84.86998 & 84.2723 & 359.17833 & 466 & 0.5 & 18 & 27 \\
\hline category6 & 423 & 0 & 177 & 0 & 70.5 & 100 & 82.69795 & 423 & 423 & 0 & 1 & 1 \\
\hline
\end{tabular}




\begin{tabular}{|c|c|c|c|c|c|c|c|c|c|c|c|c|} 
category7 & 405 & 18 & 110 & 67 & 78.64078 & 95.74468 & 86.35394 & 405.11167 & 472 & 0.1 & 38 & 53 \\
\hline category8 & 423 & 0 & 177 & 0 & 70.5 & 100 & 82.69795 & 423 & 423 & 0 & 1 & 1 \\
\hline category9 & 406 & 17 & 94 & 83 & 81.2 & 95.98109 & 87.974 & 406.13833 & 489 & 0.4 & 33 & 45 \\
\hline category10 & 403 & 20 & 52 & 125 & 88.57143 & 95.27187 & 91.79954 & 403.20833 & 528 & 0.2 & 87 & 113 \\
\hline
\end{tabular}

Here out of 600 data are used for training in both the C4.5 and CART method. Category 1, 2,3,10 is better for correctly classified instances out of 600 data during train phase. The categories $4,6,7,8$ shows that the false positive rate is large compared to other categories. Categories 6,8,11shows all data are true positive so, there will be loss if the banks take true negative data as good one.The precision , accuracy is higher for category 1,2,5,10 compared to other categories.

Table 2: CART train

\begin{tabular}{|c|c|c|c|c|c|c|c|c|c|c|c|c|}
\hline \multirow{2}{*}{ Attributes } & \multicolumn{4}{|c|}{ Confusion Matrix } & \multirow{2}{*}{ Precision } & \multirow{2}{*}{ Recall } & \multirow{2}{*}{ F_score } & \multirow{2}{*}{ Accuracy } & \multirow{2}{*}{$\mathrm{CCI}$} & \multirow{2}{*}{ Time } & \multirow{2}{*}{$\begin{array}{c}\text { No of } \\
\text { leaf }\end{array}$} & \multirow{2}{*}{$\begin{array}{c}\text { Size of } \\
\text { tree }\end{array}$} \\
\hline & TP & FN & FP & $\mathrm{TN}$ & & & & & & & & \\
\hline category1 & 389 & 34 & 91 & 86 & 91.962175 & 91.962175 & 91.962175 & 389.14333 & 475 & 2.78 & 6 & 11 \\
\hline category2 & 399 & 24 & 97 & 80 & 94.326241 & 94.32624 & 91.96217494 & 399.13333 & 479 & 1.61 & 7 & 13 \\
\hline category 3 & 389 & 34 & 91 & 86 & 91.962175 & 91.96217 & 94.32624113 & 389.14333 & 475 & 3.22 & 6 & 11 \\
\hline category4 & 417 & 6 & 149 & 28 & 98.58156 & 98.58156 & 91.96217494 & 417.04667 & 445 & 0.42 & 3 & 5 \\
\hline category5 & 411 & 12 & 111 & 66 & 97.163121 & 97.16312 & 98.58156028 & 411.11 & 477 & 1.09 & 12 & 23 \\
\hline category6 & 423 & 0 & 177 & 0 & 100 & 100 & 97.16312057 & 423 & 423 & 0.69 & 1 & 1 \\
\hline category7 & 423 & 0 & 177 & 0 & 100 & 100 & 100 & 423 & 423 & 1.05 & 1 & 1 \\
\hline category 8 & 423 & 0 & 177 & 0 & 100 & 100 & 100 & 423 & 423 & 0.66 & 1 & 1 \\
\hline category9 & 398 & 25 & 90 & 87 & 94.089835 & 94.08983 & 100 & 398.145 & 485 & 1.39 & 9 & 17 \\
\hline category10 & 399 & 24 & 97 & 80 & 94.326241 & 94.32624 & 94.08983452 & 399.13333 & 479 & 1.59 & 7 & 13 \\
\hline category11 & 423 & 0 & 177 & 0 & 100 & 100 & 94.32624113 & 423 & 423 & 0.44 & 1 & 1 \\
\hline
\end{tabular}

From the above table correctly classified instance out of 600 instances is higher in categories $9,2,10$ in the case of CART.in confusion matrix category $6,7,8,11$ shows the worst case as false positives are 177 and true positive values are 423 for all these categories.

Table 3: Naive Bayes train

\begin{tabular}{|c|c|c|c|c|c|c|c|c|c|c|}
\hline \multirow[b]{2}{*}{ Attributes } & \multirow{2}{*}{\multicolumn{4}{|c|}{ Confusion Matrix }} & \multirow[b]{2}{*}{ Precision } & \multirow[b]{2}{*}{ Recall } & \multirow[b]{2}{*}{ F_Score } & \multirow[b]{2}{*}{ Accuracy } & \multirow[b]{2}{*}{ CCI } & \multirow[b]{2}{*}{ Time } \\
\hline & & & & & & & & & & \\
\hline category1 & 375 & 48 & 82 & 95 & 82.05689 & 88.65248 & 85.22727 & 78.33333 & 470 & 0.02 \\
\hline category2 & 383 & 40 & 94 & 83 & 80.2935 & 90.54374 & 85.11111 & 77.66667 & 466 & 0 \\
\hline category3 & 375 & 48 & 82 & 95 & 82.05689 & 88.65248 & 85.22727 & 78.33333 & 470 & 0.02 \\
\hline category4 & 393 & 30 & 117 & 60 & 77.05882 & 92.9078 & 84.24437 & 75.5 & 453 & 0.02 \\
\hline category5 & 368 & 55 & 117 & 60 & 75.87629 & 86.99764 & 81.05727 & 71.33333 & 420 & 0.03 \\
\hline category6 & 414 & 9 & 164 & 13 & 71.6263 & 97.87234 & 82.71728 & 71.16667 & 427 & 0.03 \\
\hline category7 & 388 & 35 & 128 & 49 & 75.1938 & 91.72577 & 82.64111 & 72.83333 & 437 & 0.02 \\
\hline category8 & 400 & 23 & 157 & 20 & 71.81329 & 94.56265 & 81.63265 & 70 & 420 & 0.02 \\
\hline category9 & 379 & 44 & 89 & 88 & 80.98291 & 89.59811 & 85.07295 & 77.83333 & 467 & 0.02 \\
\hline category 10 & 379 & 44 & 94 & 83 & 80.12685 & 89.59811 & 84.59821 & 77 & 462 & 0.02 \\
\hline category11 & 423 & 0 & 177 & 0 & 70.5 & 100 & 82.69795 & 70.5 & 423 & 0.02 \\
\hline
\end{tabular}

Here, out of 600 data sets the higher correctly classified instances is high in categories 1,3,i.e 470 and lower in category 5,6,8 i.e. 420,414 and 400 respectively. The accuracy is high in categories, 1,3 i.e. it is $78.3333 \%$ and lower in category 8 .FP rate is rate is high in categories $4,5,6,7,8,11$ and lower in $1,2,3,9,10$.

Table 4: C4.5 test

\begin{tabular}{|c|c|c|c|c|c|c|c|c|c|c|}
\hline \multirow{2}{*}{ Attributes } & \multicolumn{3}{|c|}{ Confusion Matrix } & \multirow{2}{*}{ Precision } & \multirow{2}{*}{ Recall } & \multirow{2}{*}{ F_score } & \multirow{2}{*}{ Accuracy } & \multirow{2}{*}{ CCI } & \multirow{2}{*}{ Time } \\
\cline { 2 - 10 } & TP & FN & FP & TN & & & & & & \\
\hline category1 & 266 & 11 & 45 & 78 & 85.5305 & 96.029 & 90.47619 & 86 & 344 & 1.89 \\
\hline category2 & 264 & 13 & 56 & 67 & 82.5 & 95.307 & 88.44221 & 82.75 & 331 & 0.75 \\
\hline category3 & 265 & 12 & 101 & 22 & 72.4044 & 95.668 & 82.42613 & 71.75 & 287 & 0.25 \\
\hline category4 & 236 & 41 & 65 & 58 & 78.4053 & 85.199 & 81.6609 & 73.5 & 294 & 0.22 \\
\hline category5 & 269 & 8 & 96 & 27 & 73.6986 & 97.112 & 83.80062 & 74 & 296 & 0.28 \\
\hline category6 & 277 & 0 & 123 & 0 & 69.25 & 100 & 81.83161 & 69.25 & 277 & 0.45 \\
\hline category7 & 277 & 0 & 123 & 0 & 69.25 & 100 & 81.83161 & 69.25 & 277 & 0.45 \\
\hline category8 & 277 & 0 & 12 & 0 & 95.8478 & 100 & 97.87986 & 95.847751 & 277 & 0.22 \\
\hline category9 & 257 & 20 & 62 & 61 & 80.5643 & 92.78 & 86.24161 & 79.5 & 318 & 0.5 \\
\hline category10 & 267 & 10 & 62 & 61 & 81.155 & 96.39 & 88.11881 & 82 & 328 & 0.86 \\
\hline category11 & 277 & 0 & 123 & 0 & 69.25 & 100 & 81.83161 & 69.25 & 277 & 0.11 \\
\hline
\end{tabular}


Here out of 400 data are used for testing in both the C4.5 and CART method. The categories 6, 7,8,11 are not good for attributes for classification as there precision $69 \%$ only. The category 8 shows the best as its precision and accuracy is $95 \%$.

Table 5: CART test

\begin{tabular}{|c|c|c|c|c|c|c|c|c|c|c|}
\hline \multirow{2}{*}{ Attributes } & \multicolumn{3}{|c|}{ Confusion Matrix } & \multirow{2}{*}{ Precision } & \multirow{2}{*}{ Recall } & \multirow{2}{*}{ F_score } & \multirow{2}{*}{ Accuracy } & \multirow{2}{*}{ CCI } & \multirow{2}{*}{ Time } \\
\cline { 2 - 12 } & TP & FN & FP & TN & & & & & & \\
\hline category1 & 244 & 33 & 48 & 75 & 83.5616 & 88.08664 & 85.7645 & 79.75 & 319 & 1.89 \\
\hline category2 & 259 & 18 & 62 & 61 & 80.6854 & 93.50181 & 86.62207 & 80 & 320 & 0.75 \\
\hline category3 & 277 & 0 & 123 & 0 & 69.25 & 100 & 81.83161 & 69.25 & 277 & 0.25 \\
\hline category4 & 238 & 39 & 56 & 67 & 80.9524 & 85.92058 & 83.36252 & 76.25 & 305 & 0.22 \\
\hline category5 & 260 & 17 & 80 & 43 & 76.4706 & 93.86282 & 84.27877 & 75.75 & 303 & 0.28 \\
\hline category6 & 277 & 0 & 123 & 0 & 69.25 & 100 & 81.83161 & 69.25 & 277 & 0.45 \\
\hline category7 & 277 & 0 & 123 & 0 & 69.25 & 100 & 81.83161 & 69.25 & 277 & 0.45 \\
\hline category8 & 277 & 0 & 123 & 0 & 69.25 & 100 & 81.83161 & 69.25 & 277 & 0.22 \\
\hline category9 & 246 & 31 & 55 & 68 & 81.7276 & 88.80866 & 85.12111 & 78.5 & 314 & 0.5 \\
\hline category10 & 259 & 18 & 62 & 61 & 80.6854 & 93.50181 & 86.62207 & 80 & 320 & 0.86 \\
\hline category11 & 277 & 0 & 123 & 0 & 69.25 & 100 & 81.83161 & 69.25 & 277 & 0.11 \\
\hline
\end{tabular}

In the above table category $3,6,7,8,11$ shows higher false positive values so these are the worst attributes while category $2,5,10$ are the best categories. Category 5 consists of only4 attributes.

Table 6: Comparison of accuracy for C4.5, CART and Naïve Bayes

\begin{tabular}{|c|c|c|c|c|c|c|c|c|c|c|}
\hline \multirow{2}{*}{ Attributes } & \multicolumn{4}{|c|}{ Confusion Matrix } & \multirow{2}{*}{ Precision } & \multirow{2}{*}{ Recall } & \multirow{2}{*}{ F_score } & \multirow{2}{*}{ Accuracy } & \multirow{2}{*}{ CCI } & \multirow{2}{*}{ Time } \\
\hline & TP & $\mathrm{FN}$ & FP & $\mathrm{TN}$ & & & & & & \\
\hline category1 & 237 & 40 & 49 & 74 & 82.86713 & 85.55957 & 84.19183 & 77.75 & 311 & 0.06 \\
\hline category2 & 240 & 37 & 61 & 62 & 79.73422 & 86.6426 & 83.04498 & 75.5 & 302 & 0.03 \\
\hline category3 & 248 & 29 & 97 & 26 & 71.88406 & 89.53069 & 79.74277 & 68.5 & 274 & 0 \\
\hline category4 & 246 & 31 & 81 & 42 & 75.22936 & 88.80866 & 81.45695 & 72 & 288 & 0 \\
\hline category5 & 254 & 23 & 88 & 35 & 74.26901 & 91.69675 & 82.06785 & 72.25 & 289 & 0 \\
\hline category6 & 266 & 11 & 111 & 12 & 70.55703 & 96.02888 & 81.34557 & 69.5 & 278 & 0 \\
\hline category7 & 254 & 23 & 94 & 29 & 72.98851 & 91.69675 & 81.28 & 70.75 & 283 & 0 \\
\hline category 8 & 264 & 13 & 111 & 12 & 70.4 & 95.30686 & 80.9816 & 69 & 276 & 0 \\
\hline category9 & 240 & 37 & 63 & 60 & 79.20792 & 86.6426 & 82.75862 & 75 & 300 & 0 \\
\hline category 10 & 238 & 39 & 59 & 64 & 80.13468 & 85.92058 & 82.92683 & 75.5 & 302 & 0.02 \\
\hline category11 & 277 & 0 & 123 & 0 & 69.25 & 100 & 81.83161 & 69.25 & 277 & 0 \\
\hline
\end{tabular}

Here, from the above figure we can see the accuracy is higher in $\mathrm{C} 4.5$ for the category in comparison to CART and Naïve Bayes. The category 4 performed good because it contains only four attributes and its accuracy is higher the category 6,7,11 are the worst ones and accuracy is same in C4.5, CART and Naïve Bayes.

Table 7: Comparison of correctly classified instances for C4.5, CART and Naïve Bayes

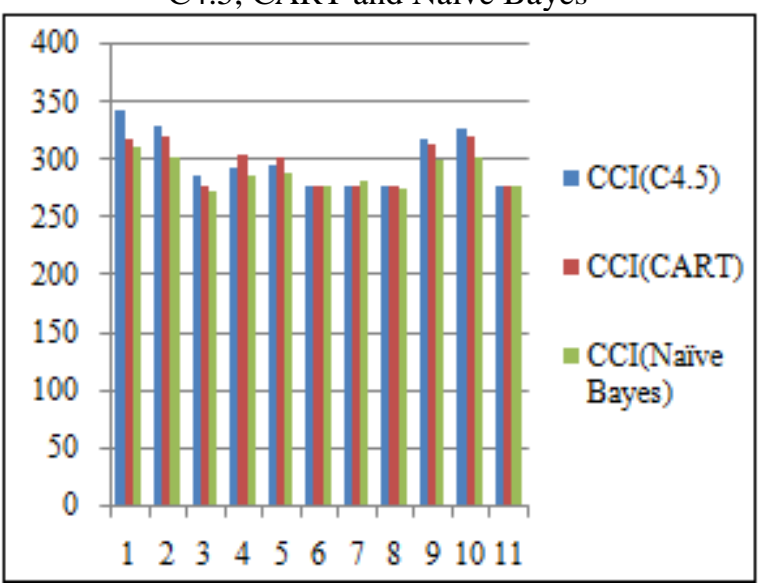

We can see the CCI using Naïve Bayes is remarkably higher compared to C4.5 and CART the categories 1,4,9,10 are good ones while category 5,11 are the worst ones.

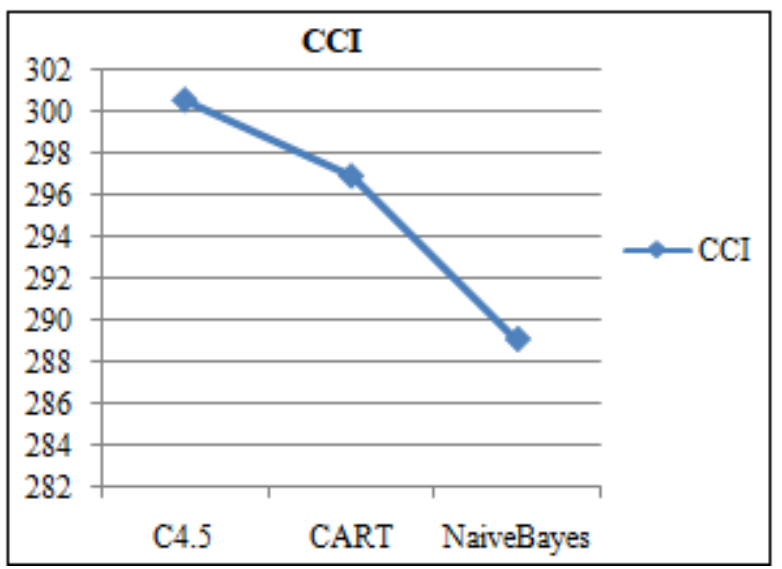

Figure 2: Average value of correctly classified instance for C4.5, CART and Naïve Bayes

From the above figure, the accuracy is higher in $\mathrm{C} 4.5$ compared to classifier Naïve Bayes and CART. 


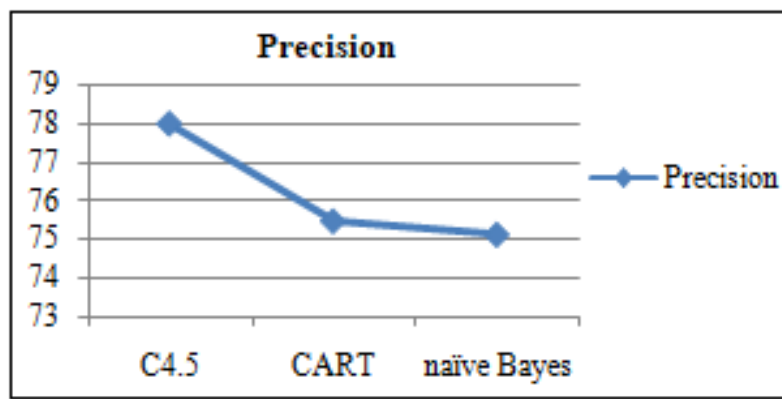

Figure 3: Average Precision value for C4.5, CART and Naïve Bayes

The average precision is remarkably higher in $\mathrm{C} 4.5$ compared to CART and Naïve Bayes. The average precision of C4.5 is 78\%, CART is 75.5 and Naïve Bayes is 75.1.

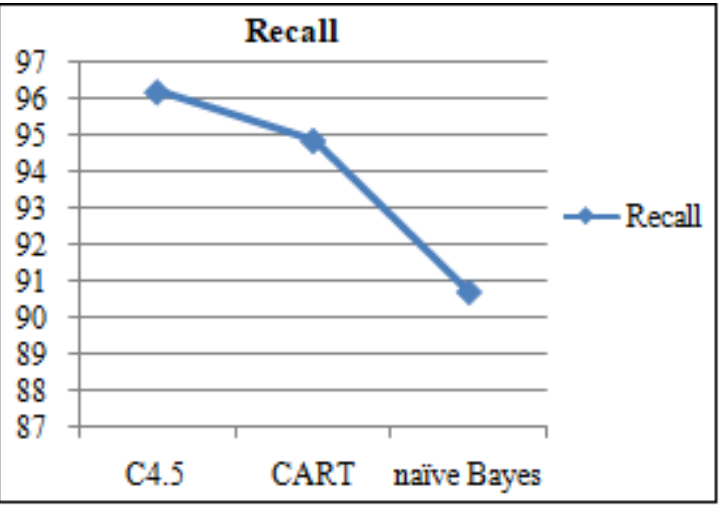

Figure 4: Average Recall value for C4.5, CART and Naïve Bayes

The average recall value is higher it is $96 \%$, CART is $95 \%$ and Naïve Bayes is $90.80 \%$ Here C4.5 is better in comparison to CART and Naïve Bayes.

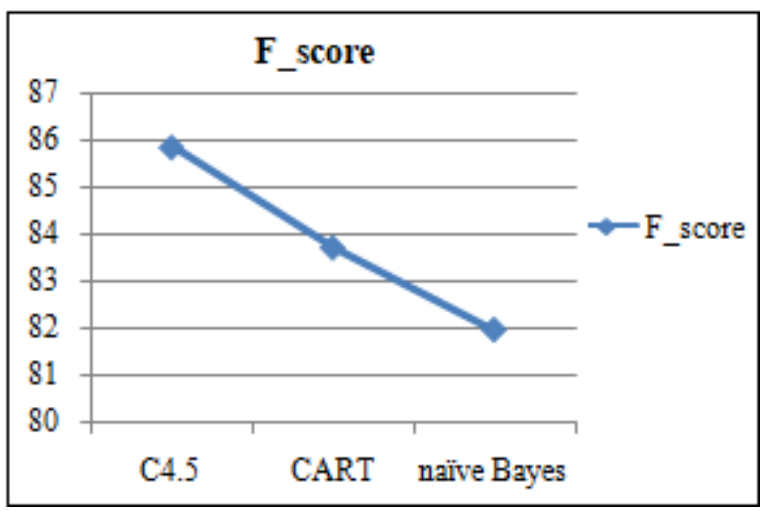

Figure 5: Average $F_{-}$score value for C4.5, CART and Naïve Bayes

The average F_score is higher in C4.5 i.e. $86 \%$, CART is $83.80 \%$ and that of Naïve Bayes is $82 \%$. Therefore we can conclude that $\mathrm{C} 4.5$ is better.

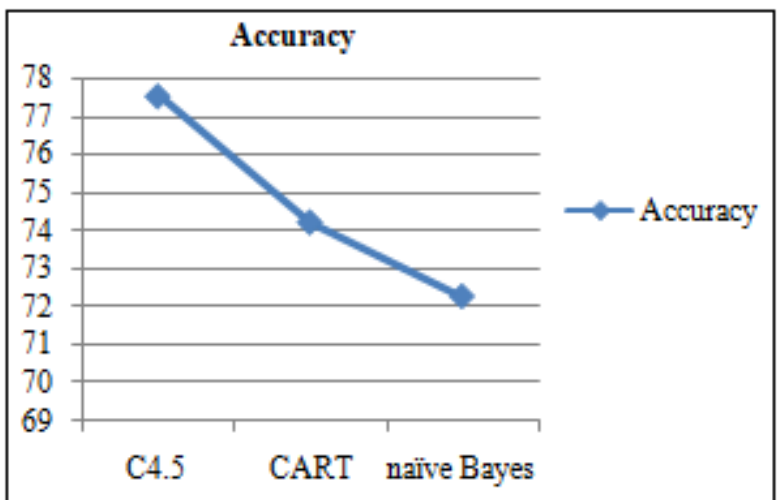

Figure 6: Average F_score value for C4.5, CART and Naïve Bayes

The average accuracy is higher in comparison to the classifier C4.5 than CART and Naïve Bayes. The average accuracy for $\mathrm{C} 4.5$ is $77.5 \%$, CART is $74 \%$ and Naïve Bayes is $72.1 \%$.

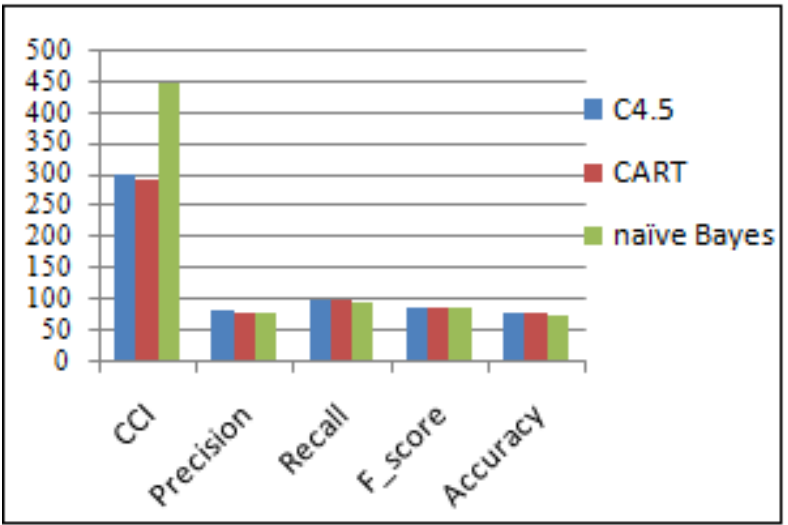

Figure 7: Collective comparison of CCI, Precision, Recall, F_score and Accuracy for C4.5, CART and Naïve Bayes

Naïve Bayes predicted higher compared to CART and C4.5 in Correctly classified instances. The average precision, recall, F_score, accuracy is high in C4.5 compared to CART and Naïve Bayes.

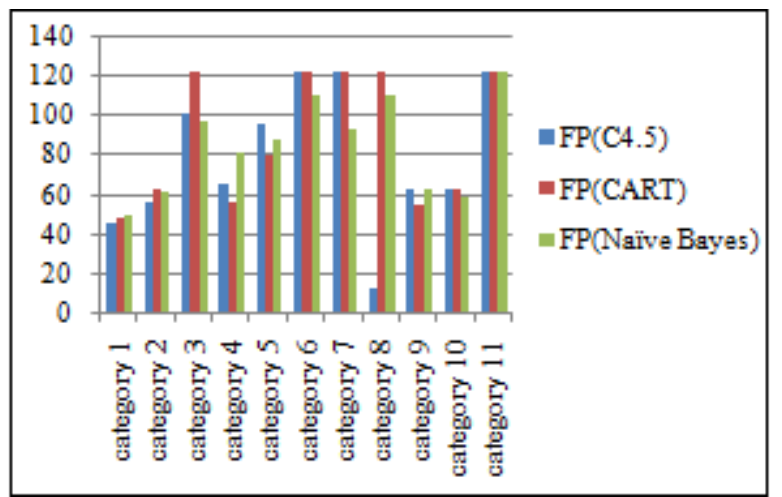

Figure 8: False positive value of Loan data for C4.5, CART and Naïve Bayes

The main focus is on the false positive value as it is the positive count for the bad customers that it the most risk factor for the loan prediction. The categories $1,2,4,8,9,10$ contain the lower FP value in which in category 8. C4.5 has the lowest FP. The category 4 is also acceptable as it contains only 4 attributes in which FP is low. The categories $3,6,7,11$ are the worst one. 


\section{Conclusion and Discussion}

If a customer with bad credit is misclassified as a customer with good credit then a bank will suffer. In this research three different classifiers, C4.5, CART and Naïve Bayes have been applied to predict loan grants and the attribute selection in them. More, financial institution is seeking better strategies through the help of credit scoring models. Therefore, it is concluded that categories 4,8 is the best one and categories 3,6,11 are the worst as it counts false positive value is greater in all the C4.5,CARTand Naïve Bayes testing. Among the classifier C4.5, CART and Naïve Bayes, $\mathrm{C} 4.5$ is the best classifier to predict loan.

\section{References}

[1] Pratik Gosar,Paras Kapadia , Niharika, Maheswori, Pramila Chawan K. Chopde, "A study of a Classification Based Credit Risk Analysis Algorith," Intenational Journal of Engineering and Advanced Technology, vol. 1, no. 2249-8958, p. 3, April 2012.

[2] Sarika Chaudary Sanjay Kumar Maliki, "Comparative Study of Decision Tree Algorithms For Data Analysis," International Journal of Research in Computer Engineering and Electronics, p. 8, June2013.

[3] A.Yilmaz camurcu Serhat Ozekes, "Classification and Prediction in a Data Mining Application," Journal of Marmarafor Pure Applied Science, pp. 169-174, 2002.

[4] Yu,Zhong Xiao-Lin, "An Overview of Personal Credit Scoring:Techniques and Future Work," International Journal of Intelligence Science, pp. 181-189, August 2012.

[5] Daniela Schiopu Irina Ionita, "Usig Principal Component Analysis in Loan Granting," pp. 88-96, 2010.

[6] Bora Aktan Husey Incea, "A Comparison of data Mining Techniques For Credit Scoring In Banking:A Managerial Perspective," Journal of Business Economics and Management, pp. 233-240, march 2009. [1]

[7] Arun K.Pujari, Data Mining Techniques. Hyderabad, India: Universities press private limited.

[8] P.-N. T.-N. T. Pang-Ning Tan. [Online]. Available: https://wwwusers.cs.umn.edu/ kumar001/dmbook/sol. pdf.

[9] P. D. H. Hofmann. [Online]. Available http://archive.ics.uci.edu/ml/datasets/Statlog+\%28Ger man+Credit+Data\%29

[10] P. T. A. T. Kalyani R. Rawate, "Review on predication system for bank loan credibility," Scientific Journal of Impact Factor (SJIF): 4.72, vol. 4, no. 12, 2017.

[11] P. C. Abhijit A. Sawant, "Comparison of Data Mining Techniques used for Financial," International Journal of Emerging Technology and Advanced Engineering, vol. 3 , no. 6 , p. 6, 2013. 